\title{
A mini-review of TAT-MyoD fused proteins: state of the art and problems to solve
}

\author{
Marco Patruno (1), Luca Melotti (1), Chiara Gomiero (1), Roberta Sacchetto (1), Ohad \\ Topel (2), Tiziana Martinello (1)
}

(1) Department of Comparative Biomedicine and Food Science, University of Padova, Italy; (2) VTH - Koret School of Veterinary Medicine, The Robert H. Smith Faculty of Agriculture, Food and Environment, The Hebrew University of Jerusalem, Israel

This article is distributed under the terms of the Creative Commons Attribution Noncommercial License (CC BY-NC 4.0) which permits any noncommercial use, distribution, and reproduction in any medium, provided the original author(s) and source are credited.

\begin{abstract}
The transcriptional activator TAT is a small peptide essential for viral replication and possesses the property of entering the cells from the extracellular milieu, acting as a membrane shuttle. In order to safely differentiate cells an innovative methodology, based on the fusion of transcription factors and the TAT sequence, is discussed in this short review. In several studies, it has been demonstrated that TAT protein can be observed in the cell nucleus after few hours from the inoculation although its way of action is not fully understood. However, further studies will be necessary to develop this methodology for clinical purposes.
\end{abstract}

Key Words: Tat-MyoD, C2C12, PB-MSCs, coculture, myogenic induction

Eur J Transl Myol 27 (4): 234-238

The HIV-1 trans-activator gene (TAT) is essential for the latent transcription of the HIV virus, for its replication and for its gene expression. ${ }^{1}$ In addition, the TAT protein is able to conjugate with exogenous molecules (like nucleic acids, protein, peptides or drug molecules) mediating their delivery into the cell through the plasma membrane or the nucleus through the nuclear membrane., ${ }^{2,3}$ As a matter of fact, the TAT protein belongs to the protein transduction domain (PTD) family. Its ability to fuse and deliver recombinant proteins, as well as therapeutic ones, has been studied in different fields (e.g. disease treatment and diagnosis), especially in the research for the cancer treatment. ${ }^{4}$ The HIV TAT protein transduction motif along with the Drosophila antennapeptide (Antp) and the herpes simplex virus VP22 protein, are generally the most studied PTDs. They differ in their length and amino acid sequence but they share the same "cell-penetrating effect". 5 There are several reasons why PTDs are widely used: they transport macromolecules into cells, they have almost no cytotoxicity and most importantly they usually do not modify the genome of the target cells. These characteristics give the PTDs an excellent biological safety; its transduction ability and characteristics appear interesting to induce tissuespecific differentiation when the TAT protein is associated to transcription factors, e.g. in the myogenic differentiation, ${ }^{6}$ or in reprogramming adult stem cells. ${ }^{7}$
The purpose of this short review was to summarize the principal characteristics of TAT and its role in the differentiation or reprogramming when conjugated to particular proteins.

\section{Structure, functional domain and mechanism of uptake}

The HIV-1 TAT protein is 86 amino acids long and consists of two exons: the first one of 72 amino acids and the second of $14 .^{8}$ According to its amino acidic sequence this protein can be divided into different domains, the most important one is the core domain (aa $47-57)^{9,10}$ in which relies the transducing ability of the TAT protein. The amino acidic composition of the core domain is fundamental to carry out the transduction of the cargo into target cells. Some studies have shown that to enable an efficient transmembrane movement this domain must be rich in basic amino acids (like arginine or lysine). In fact, an enrichment in positively charged amino acids enhances its function. ${ }^{11}$ This led researchers to improve the efficiency of the transduction of TAT by modifying its sequence adding basic amino acids or by modification of its structure. ${ }^{12,13}$ Indeed, it has been demonstrated that short peptides rich in arginine are rapidly internalized by cells, in a receptor-independent manner; ${ }^{8,14}$ therefore, it was suggested that the process of TAT internalization occurs through adsorptive endocytosis. This does not happen for TAT basic domain when fused to protein cargos. ${ }^{15}$ 


\section{TAT usage for iPSCs generation}

Induced pluripotent stem cells (iPSCs) ${ }^{16}$ might be a useful tool for disease modeling as well as in regenerative medicine. However, before the clinical application of this method, the safety and efficiency must be completely assessed. At first, in order to generate iPSCs, the reprogramming procedure was accomplished with genome-integrating retroviruses but this may lead to an unwanted genetic dysfunction (e.g. mutagenesis) and this is unlikely to be accepted in a clinical scenario. ${ }^{17,18}$ As a result, more virus-free strategies were developed to overcome the genetic manipulation: Sendai virus, ${ }^{19}$ episomal vectors ${ }^{20}$ or plasmids. ${ }^{21}$ Albeit a reduction in the genome integration was observed by these approaches, the DNA excised from the vectors can lead to transgene insertion. Consequently, a technique which does not involve the usage of nucleic acid was desirable. The delivery of recombinant proteins using PTDs is a possible way to overcome the issue of the DNA mutagenesis and it seems to be the most suitable and effective one. ${ }^{22}$ This delivery method guarantees no genomic integration but on the other hand it shows a relatively lower efficiency than the previous methods. Therefore, it was necessary to study methods, like the small chemical molecules, that can support the protein-based iPSCs generation order to improve its limited reprogramming ability, e.g. Valproic acid (VPA). ${ }^{23}$ Zhou et al. (2009) ${ }^{24}$ showed for the first time the generation of mouse iPSCs using a 11 arginine (R)-tagged recombinant protein combined with the histone deacetylase (HDAC) inhibitor VPA. A successful generation of iPSCs in human newborn fibroblast was obtained by Kim et al. (2009) ${ }^{25}$ using a 9arginine (9R) fused to the reprogramming factors (RF) Oct-4, Sox-2, Klf4 and c-Myc. In a later study, Zhang et al. $(2012)^{26}$ compared the latter delivery approach and TAT-recombinant protein in iPSCs reprogramming in human foreskin fibroblasts. Their outcomes showed that RF fused with TAT resulted in a higher transcriptional activity.

\section{TAT-MyoD fused protein: an example of TAT} application in an in vitro differentiation protocol

MyoD is a transcription factor that plays a key role in the muscular differentiation. ${ }^{27}$ In addition, Hidema et al. $(2012)^{28}$ showed that MyoD protein fused with TAT has a higher differentiating potential than the wild type MyoD. Recently, the potential of the complex TATMyoD in inducing equine peripheral blood mesenchymal stem cells (PB-MSCs) towards the myogenic fate has been evaluated. ${ }^{6}$ Mesenchymal stem cells are an undifferentiated multipotent cell population which deserves a particular attention because they offer an alternative therapeutic solution for muscle diseases. ${ }^{29}$ Up to now, scarce data were present in literature about the differentiation of MSCs into myoblasts, but in vitro it has been shown that MSCs may differentiate into skeletal muscle cells with conditioned medium as well as in co-colture with a fusion between MSCs and myoblasts. ${ }^{30-32}$ Although data indicate that TAT-MyoD induces myogenic differentiation in naturally predisposed cells only, like the $\mathrm{C} 2 \mathrm{C} 12$ cell line ${ }^{33}$ or the mouse muscle primary cells, ${ }^{28}$ some authors demonstrated that the efficiency of myogenic differentiation with Tat-MyoD transduction of human adipose-derived stem cells was reached only when it was fused with $\mathrm{C} 2 \mathrm{C} 12$ myoblasts. ${ }^{31}$ Our research group has shown that the supplement of TAT-MyoD alone was not sufficient to induce cellular differentiation, even if it activated the myogenic pathway at the nuclear level. Therefore, in order to achieve the myogenic differentiation of MSCs, conditioned medium was added. This creates a suitable in vitro microenvironment for the differentiation towards muscle cells. These results suggest that TAT-mediated protein transduction system, if supported by a conditioned medium, might represents a useful methodology to induce myoblasts differentiation. Moreover, this indicates that the development of myogenic phenotypes of mesenchymal stem cells by TAT-MyoD construct depends on time and culture conditions, highlighting the essential role of the in vitro microenvironment in terms of secreted factors and cell contacts; other studies confirmed the necessity to have other factors apart from the genetic ones (i.e. MyoD) to commit undifferentiated cells. ${ }^{34-37}$ The advantage of having a robust myogenic differentiation method by means of the TAT-mediated protein transduction consists in obtaining committed myogenic cells derived from an abundant cell source, like the peripheral blood, without the need to fuse them with other cells. Certainly, this innovative approach of protein transduction with TAT fused with various transcription factors appears extremely interesting in the therapeutic and regenerative medicine field. ${ }^{38-41}$

\section{Limitations and possible solutions}

Even though progress has been made on TAT transduction ability for research purposes there are still some intriguing issues, e.g. slow endosomal release. In fact, if the TAT-recombinant protein complex is packaged for too long in the endosomes it could be degraded or refolded. ${ }^{42}$ However, scientists tried to overcome this problem with the use of chemical auxiliary like $\mathrm{Ca}^{2+}$ or sucrose, ${ }^{43}$ the use of radiation after labeling the TAT protein with photosensitive agents ${ }^{44}$ or the modification of its amino acidic sequence as previously stated. Another concern about the usage of TAT is the quality and the production of the recombinant protein to which TAT is fused. Even if these proteins are usually produced in a highly efficient way with bacterial expression vectors ${ }^{45}$ the real issues are the consequential steps of purification that may affect the biological activity of the protein. ${ }^{46}$ The expression in the mammalian cells might be an alternative to the bacterial expression system, in order to 
have proper post-translational modifications such as a correct folding of the protein. ${ }^{47}$ In the future, the research should focus on the study of the mechanism of internalization because it is still unclear although the internalization by macropinocytosis it is the most validated hypothesis. ${ }^{48}$ Identifying new small chemical compounds that can enhance the transduction is another goal due to its low efficiency in reprogramming target cells. Moreover, its application in in vivo study should be more investigated. First of all, it would be necessary to produce an amount of secreted protein sufficient for large-scale studies. Then it should be performed a research about its possible toxicity in vivo. Although it has been widely demonstrated that the HIV-1 TAT protein can deliver many molecules like peptides, proteins, drug molecules or nucleic acids more in vivo studies are mandatory. In conclusion, thanks to recent experiments it has been also assessed that it has an excellent biological safety; these characteristics, plus the absence of modification of the genetic code, make this method a suitable tool for different fields of study such as pharmacology, disease treatment and direct lineage reprogramming for regenerative purposes. ${ }^{49}$

Combined with recent advancements in skeletal muscle clinical modulation and regeneration, ${ }^{50-54}$ the described innovative methodology based on the fusion of transcription factors and the TAT sequence, may open new options for patients in need of skeletal muscle reconstruction.

\section{List of acronyms}

TAT - Trans-activator of transcription

PTD - Protein transduction domain

Antp - Antennapeptide

HDAC - Histone deacetylase

VPA - Valproic acid

iPSCs - induced Pluripotent stem cells

RF - Reprogramming factors

MyoD - Myogenic differentiation antigen

PB-MSCs - Peripheral blood mesenchymal stromal cells

\section{Author's contributions}

M. Patruno and T. Martinello participated in experimental design and writing; L. Melotti, O. Topel and C. Gomiero contributed to data collection.

R. Sacchetto participated in the revision of the manuscript.

\section{Acknowledgments}

This work was supported by a grant from the University of Padova, Italy (PRAT 2013, code number CPDA138242).

\section{Conflict of Interest}

None of the authors have conflicts of interests.

\section{Ethical Publication Statement}

We confirm that we have read the Journal's position on issues involved in ethical publication and affirm that this report is consistent with those guidelines.

\section{Corresponding Author}

Marco Patruno, Department of Comparative Biomedicine and Food Science, University of Padova, Italy .E-mail: marco.pat@unipd.it

\section{E-mails of co-author}

Luca Melotti: luca.melotti.4@phd.unipd.it Chiara Gomiero: chiara.gomiero@unipd.it Roberta Sacchetto: roberta.sacchetto@unipd.it Ohad Topel: ohadtopel@gmail.com

Tiziana Martinello: tiziana.martinello@unipd.it

\section{References}

1. Karn J. The molecular biology of HIV latency: breaking and restoring the Tat-dependent transcriptional circuit. Curr Opin HIV AIDS 2011;6:4-11.

2. Mäe M, Langel U. Cell-penetrating peptides as vectors for peptide, protein and oligonucleotide delivery. Curr Opin Pharmacol 2006;6:509-14.

3. Milletti F. Cell-penetrating peptides: classes, origin, and current landscape. Drug Discov Today 2012;17:850-60.

4. Wadia J, Dowdy S. Transmembrane delivery of protein and peptide drugs by TAT-mediated transduction in the treatment of cancer. Adv Drug Deliv Rev 2005;57:579-96.

5. Schwarze SR, Hruska KA, Dowdy SF. Protein transduction: unrestricted delivery into all cells?. Trends Cell Biol 2000;10:290-5.

6. Patruno M, Gomiero C, Sacchetto R, et al. TatMyoD fused proteins, together with $\mathrm{C} 2 \mathrm{C} 12$ conditioned medium, are able to induce equine adult mesenchimal stem cells towards the myogenic fate. Vet Res Commun 2017;41:211-7.

7. Dai Y, Guo Y, Wang C, et al. Non-genetic direct reprogramming and biomimetic platforms in a preliminary study for adipose-derived stem cells into corneal endothelia-like cells. PLoS One 2014;9:e109856.

8. Green, M, Loewenstein PM. Autonomous functional domains of chemically synthesized human immunodeficiency virus Tat trans-activator protein. Cell 1988;55:1179-88.

9. Mann DA, Frankel AD. Endocytosis and targeting of exogenous HIV-1 Tat protein. EMBO J 1991;10:1733-9.

10. Vivès, E, Brodin P, Lebleu B. A truncated HIV-1 tat protein basic domain rapidly translocates through the plasma membrane and accumulates in the cell nucleus. J Biol Chem 1997;272:16010-7.

11. Rothbard JB, Garlington S, Lin Q, et al. Conjugation of arginine oligomers to cyclosporin 
A facilitates topical delivery and inhibition of inflammation. Nat Med 2000;6:1253-7.

12. Wender PA, Mitchell DJ, Pattabiraman K, et al. The design, synthesis, and evaluation of molecules that enable or enhance cellular uptake: peptoid molecular transporters. Proc Natl Acad Sci U S A 2000;97:13003-8.

13. Ho A, Schwarze SR, Mermelstein SJ, et al. Synthetic protein transduction domains: enhanced transduction potential in vitro and in vivo. Cancer Res 2001;61:474-7.

14. Suzuki T, Futaki S, Niwa M, et al. Possible existence of common internalization mechanisms among arginine-rich peptides. J Biol Chem 2002;277:2437-43.

15. Fittipaldi A, Giacca M. Transcellular protein transduction using the Tat protein of HIV-1. Adv Drug Deliv Rev 2005;57:597-608.

16. Takahashi K, Yamanaka S. Induction of pluripotent stem cells from mouse embryonic and adult fibroblast cultures by defined factors. Cell 2006;126:663-76.

17. Okita K, Ichisaka T, Yamanaka S. Generation of germline-competent induced pluripotent stem cells. Nature 2007;448:313-7.

18. Yamanaka S. Strategies and new developments in the generation of patient-specific pluripotent stem cells. Cell Stem Cell 2007;1:39-49.

19. Fusaki N, Ban H, Nishiyama A, et al. Efficient induction of transgene-free human pluripotent stem cells using a vector based on sendai virus, an RNA virus that does not integrate into the host genome. Proc Jpn Acad Ser B Phys Biol Sci 2009;85:348-62.

20. Yu J, Hu K, Smuga-Otto K, et al. Human induced pluripotent stem cells free of vector and transgene sequences. Science 2009;324:797-801.

21. Okita K, Hong H, Takahashi K, Yamanaka S. Generation of mouse-induced pluripotent stem cells with plasmid vectors. Nat Protoc 2010;5:41828.

22. Ziegler A, Seelig J. High affinity of the cellpenetrating peptide HIV-1 Tat-PTD for DNA. Biochemistry 2007;46:8138-45.

23. Huangfu D, Maehr R, Guo W, et al. Induction of pluripotent stem cells by defined factors is greatly improved by small-molecule compounds. Nature Biotechnology 2008;26:795-7.

24. Zhou H, Wu S, Joo JY, et al. Generation of induced pluripotent stem cells using recombinant proteins. Cell Stem Cell 2009;4:381-4.

25. Kim D, Kim CH, Moon JI, et al. Generation of human induced pluripotent stem cells by direct delivery of reprogramming proteins. Cell Stem Cell 2009;4:472-6.

26. Zhang H, Ma Y, Gu J, et al. Reprogramming of somatic cells via TAT-mediated protein transduction of recombinant factors. Biomaterials 2012;33:5047-55.

27. Davis, R L, H Weintraub, and A B Lassar. Expression of a single transfected cDNA converts fibroblasts to myoblasts. Cell 1987;51:987-1000.

28. Hidema S, Tonomura Y, Date S, Nishimori K. Effects of protein transduction with intact myogenic transcription factors tagged with HIV-1 Tat-PTD (T-PTD) on myogenic differentiation of mouse primary cells. J Biosci Bioeng 2012;113:511.

29. Mizuno H. 2010. The Potential for Treatment of Skeletal Muscle Disorders with Adipose-Derived Stem Cells. Curr Stem Cell Res Ther 2010;5:1336.

30. Dezawa $\mathrm{M}$, Ishikawa $\mathrm{H}$, Itokazu $\mathrm{Y}$, et al. Bone marrow stromal cells generate muscle cells and repair muscle degeneration. Science 2005;309: 314-7.

31. Sung MS, Mun JY, Kwon O, et al. Efficient myogenic differentiation of human adiposederived stem cells by the transduction of engineered MyoD protein. Biochem Biophys Res Commun 2013;437:156-61.

32. Dugan JM, Cartmell SH, Gough JE. Uniaxial cyclic strain of human adipose-derived mesenchymal stem cells and C2C12 myoblasts in coculture. J Tissue Eng 2014;27;5:204 1731414530138.

33. Noda T, Fujino T, Mie M, Kobatake E. Transduction of MyoD protein into myoblasts induces myogenic differentiation without addition of protein transduction domain. Biochem Biophys Res Commun 2009;382:473-7.

34. Kashanchi F, Khleif SN, Duvall JF, et al. Interaction of human immunodeficiency virus type 1 TAT with a unique site of TFIID inhibits negative cofactor Dr1 and stabilizes the TFIIDTFIIA complex. J Virol 1996;70:5503-10.

35. Marzio G, Tyagi M, Gutierrez MI, Giacca M. HIV-1 Tat transactivator recruits p300 and CREBbinding protein histone acetyltransferases to the viral promoter. Proc Natl Acad Sci U S A 1998;95:13519-24.

36. Gupta A, Leong DT, Bai HF, et al. Osteomaturation of adipose-derived stem cells required the combined action of vitamin D3, betaglycerophosphate, and ascorbic acid. Biochem Biophys Res Commun 2007;362:17-24.

37. Lee JY, Zhou Z, Taub PJ, et al. BMP-12 treatment of adult mesenchymal stem cells in vitro augments tendon-like tissue formation and defect repair in vivo. PLoS One 2011;6:e17531.

38. Hua WK, Shiau YH, Lee OK, Lin WJ. Elevation of protein kinase C? stimulates osteogenic differentiation of mesenchymal stem cells through the TAT-mediated protein transduction system. Biochem Cell Biol 2013;91:443-8. 
39. Kanno H, Kubo A, Yoshizumi T, et al. Isolation of multipotent nestin-expressing stem cells derived from the epidermis of elderly humans and TATVHL peptide-mediated neuronal differentiation of these cells. Int J Mol Sci 2013;14:9604-17.

40. Lin BY, Kao MC. Therapeutic Applications of the TAT-mediated protein transduction system for complex I deficiency and other mitochondrial diseases. Ann N Y Acad Sci 2015;1350:17-28.

41. Woo SJ, Shin MJ, Kim DW, et al. Effects of low doses of Tat-PIM2 protein against hippocampal neuronal cell survival. J Neurol Sci 2015;358:22635.

42. Cai SR, Xu G, Becker-Hapak M et al. The kinetics and tissue distribution of protein transduction in mice. Eur J Pharm Sci 2006;27:311-9.

43. Qin Y, Wang GX, Liu F. Research and application of a new macro molecular nano-carrier-cell penetrating peptides. Chin J Cell Biol 2014;36:585-90.

44. Maiolo JR, Ottinger EA, Ferrer M. Specific redistribution of cell-penetrating peptides from endosomes to the cytoplasm and nucleus upon laser illumination. J Am Chem Soc 2004;126: 15376-7.

45. Nagahara, H, Vocero-Akbani AM, Snyder EL, et al. Transduction of full-length TAT fusion proteins into mammalian cells: TAT-p27Kip1 induces cell migration. Nat Med 1998;4:1449-52.

46. Singh A, Upadhyay V, Upadhyay AK et al. Protein recovery from inclusion bodies of Escherichia coli using mild solubilization process. Microb Cell Fact 2015;14: 41.
47. Hartley JL. Why proteins in mammalian cells? Methods Mol Biol 2012;801:1-12.

48. Wadia JS, Stan RV, Dowdy SF. Transducible TAT-HA fusogenic peptide enhances escape of TAT-fusion proteins after lipid raft macropinocytosis. Nature Medicine 2004;10:3105.

49. $\mathrm{Xu} J, \mathrm{Du} \mathrm{Y}$, Deng $\mathrm{H}$. Direct lineage reprogramming: strategies, mechanisms, and applications. Cell Stem Cell 2015;16:119-34.

50. Carlson BM. The Biology of Long-Term Denervated Skeletal Muscle. Eur J Transl Myol 2014;24:3293.

51. Edmunds KJ, Gargiulo P. Imaging Approaches in Functional Assessment of Implantable Myogenic Biomaterials and Engineered Muscle Tissue. Eur J Transl Myol 2015;25:4847.

52. Carraro U, Boncompagni S, Gobbo V, et al. Persistent Muscle Fiber Regeneration in Long Term Denervation. Past, Present, Future. Eur J Transl Myol 2015;25:4832.

53. Scicchitano BM, Sica G, Musarò A. Stem Cells and Tissue Niche: Two Faces of the Same Coin of Muscle Regeneration. Eur J Transl Myol 2016;26:6125.

54. Kern H, Hofer C, Loefler S, Zampieri S, et al. Atrophy, ultra-structural disorders, severe atrophy and degeneration of denervated human muscle in SCI and Aging. Implications for their recovery by Functional Electrical Stimulation, updated 2017. Neurol Res 2017;39:660-6. 